\title{
STABILITY AND BIFURCATION IN A MODULATED BURGERS SYSTEM*
}

\author{
BY \\ W. E. OLMSTEAD ${ }^{1}$ AND S. H. DAVIS ${ }^{2}$ \\ Northwestern University
}

\begin{abstract}
The stability of the null state for a nonlinear Burgers system is examined. The results include (i) an energy estimate for global stability for states involving arbitrary modulation in time, and (ii) an analysis of the bifurcation from the null state for slow modulations. For the slow modulations it is determined that the amplitude $A(\tau)$ of the bifurcated disturbance velocity satisfies a Landau-type equation with time-dependent growth rate $\theta(\tau)$. Particular attention is given to periodic and quasiperiodic modulations of the system, which lead to analogous behavior in $\theta(\tau)$. For each of these oscillatory-type modulations, it is found that $A^{2}(\tau)$ has the same long-time mean value as the unmodulated case, implying no alteration of the final mean kinetic energy. Applications to various fluid-dynamical phenomena are discussed.
\end{abstract}

1. Introduction. The system of equations

$$
\begin{gathered}
\frac{d U}{d t}=P-U-\frac{1}{R} \int_{0}^{1} u^{2}(\eta, t) d \eta, \quad t \geq 0 \\
\frac{\partial u}{\partial t}+2 u \frac{\partial u}{\partial y}=\frac{\partial^{2} u}{\partial y^{2}}+R u U, \quad 0 \leq y \leq 1, \quad t \geq 0,
\end{gathered}
$$

with

$$
u(0, t)=u(1, t)=0, \quad u(y, 0)=u_{0}(y)=\varepsilon h(y), \quad U(0)=U_{0},
$$

was proposed by Burgers [1, 2] as a model for turbulent flow in a channel. In this model $U(t)$ denotes a mean velocity driven by a pressure gradient $P$ and $R$ represents the Reynolds number. The integral quantity in Eq. (1.1a) plays the role of the Reynolds stress which governs energy conversion between the fluctuation $u(y, t)$ and the mean.

Although the above Burgers model has not proved to be a reliable estimator of shear flow turbulence, this set does incorporate the nonlinearity of the Navier-Stokes equations and has the potential of giving insight into some nonlinear behavior predicted by the physical equations of motion. Indeed, versions of this model [3] accurately represent shock formation in gases and the tendency toward breaking in surface water waves. Hence there is a good reason to study the nonlinear properties of system (1.1) in order to understand questions that seem presently intractable when posed for the Navier-Stokes equations.

* Received September 18, 1980.

${ }^{1}$ This author's work was supported by NSF MCS-8002759, DOE DE-AS02-78ER4650, and AFOSR-800016.

${ }^{2}$ This author's work was supported by ARO DAAG29-79-G-0030. 
Previous investigations $[1,2,4,5,6]$ of system (1.1) have been concerned with the stability properties of the basic solution given by

$$
\bar{U} \equiv P, \quad u \equiv 0,
$$

where $P$ is constant. Our goal here is to allow the pressure gradient $P(t)$ to be timedependent and hence examine the nonlinear stability properties of the modulated state given by

$$
d \bar{U} / d t=P(t)-\bar{U}(t), \quad u \equiv 0 .
$$

It is convenient to solve Eq. (1.1a) for $U$ and substitute into Eq. (1.1b). Thus, system (1.1) becomes an integro-differential system for $u(y, t)$, namely

$$
\frac{\partial u}{\partial t}+2 u \frac{\partial u}{\partial y}=\frac{\partial^{2} u}{\partial y^{2}}+\lambda(t) u-e^{-t} u \int_{0}^{t} \int_{0}^{1} u^{2}(\eta, s) e^{s} d \eta d s,
$$

with

$$
u(0, t)=u(1, t)=0, \quad u(y, 0)=u_{0}(y)=\operatorname{ch}(y)
$$

where $\varepsilon$ is a small parameter measuring the size of the initial data. The function $\lambda$ is defined as

$$
\lambda(t)=\operatorname{Re}^{-t}\left[U_{0}+\int_{0}^{t} e^{s} P(s) d s\right] .
$$

We shall assume in what follows that $P(t)$ is a given piecewise continuous function so that $\lambda$ is continuous.

We are concerned with the stability of the null solution $u \equiv 0$. In particular, we wish to examine the effect of slowly modulated pressure fields for which

$$
P(t)=p\left(\varepsilon^{2} t\right) .
$$

For this class of pressure fields there is the possibility that the slow variations induce an oscillation about the critical state and could even lead to shifts in the critical point. Such situations in hydrodynamic states have had much recent attention and have been surveyed by Davis [7] for cases where the modulation is time-periodic. In the present work we shall generalize the class of modulations to include both periodic and nonperiodic behaviors. We shall concentrate on the weakly-nonlinear behavior of branches and carefully examine the secondary states of the system (1.3) and (1.4) for $t \rightarrow \infty$. A first attempt at such an analysis was made by Davis [8] for a special case of Bénard convection, though he did suggest that the amplitude equations obtained had wider applicability. DiPrima and Stuart [9] considered the effect of small eccentricity on the onset of Taylor vortices and obtained a theory of similar type. The basic flow field examined was time-periodic. Hall [10] used a similar approach for time-periodic modulation of cylinders in the production of Taylor vortex flows. If one thinks of the modulation of the problem as inducing the bifurcation parameter $\lambda$ to undergo slow variations about the bifurcation point $\lambda_{c}$, then our problem is related to that of Rubenfeld [11] who considered bifurcation in a periodically modulated nonlinear heat equation. Rubenfeld obtains an amplitude equation similar to those given by Davis [8], DiPrima and Stuart [9] and Hall [10]; he also obtains some criteria of stability and instability from the amplitude equation. In the present work we obtain for the Burgers 
system an amplitude equation of similar type to those mentioned above. However, we generalize the derivation to include more general modulations and prove some new properties of this generalized amplitude equation.

Another aspect of the investigation here concerns a wider class of functions $\lambda(t)$ (and hence $P(t))$ under which stability of the null solution is guaranteed. In this class, $\lambda(t)$ is not necessarily slowly varying. We derive an energy inequality from which we can establish that

$$
\int_{0}^{1} u^{2}(y, t) d y \rightarrow 0 \text { as } t \rightarrow \infty .
$$

2. Asymptotic analysis. We seek solutions of system (1.3) and (1.4) that bifurcate from the null solution and remain bounded as $t \rightarrow \infty$. In order to represent such solutions $u(y, t)$, we will employ a formal asymptotic expansion approach using two time scales. We introduce the slow time variable

$$
\tau=\varepsilon^{2} t
$$

and assume an asymptotic expansion of the form

We also note that

$$
u(y, t) \sim \sum_{j=1} w_{j}(y, t, \tau) \varepsilon^{j} .
$$

$$
\lambda(t)=R\left\{p(\tau)+e^{-t}\left[U_{0}-p(0)+O\left(\varepsilon^{2}\right)\right]\right\},
$$

so that $\lambda(t)$ represents a shifted version of the Reynolds number $R$ or, as viewed by Rubenfeld [11], a slowly varying bifurcation parameter. This suggests the introduction of the slowly-varying function $\theta$,

$$
\theta(\tau)=\left[\lambda(t)-\lambda_{c}\right] / \varepsilon^{2},
$$

as a measure of the pressure variation about the critical value $\lambda_{c}$ associated with the bifurcation point.

We then follow the general scheme used by Matkowsky [12] to obtain a recursive system of equations for the determination of the $w_{j}$. Inserting (2.1), (2.2) and (2.4) into (1.3) and (1.4) while equating to zero coefficients of like powers of $\varepsilon$, we find for the first three orders,

$$
\begin{gathered}
\left(\partial^{2} w_{1} / \partial y^{2}\right)+\lambda_{c} w_{1}-\left(\partial w_{1} / \partial t\right)=0, \\
w_{1}(0, t, \tau)=w_{1}(1, t, \tau)=0, \quad w_{1}(y, 0,0)=h(y) ; \\
\left(\partial^{2} w_{2} / \partial y^{2}\right)+\lambda_{c} w_{2}-\left(\partial w_{2} / \partial t\right)=2 w_{1}\left(\partial w_{1} / \partial y\right), \\
w_{2}(0, t, \tau)=w_{2}(1, t, \tau)=0, \quad w_{2}(y, 0,0)=0 ; \\
\left(\partial^{2} w_{3} / \partial y^{2}\right)+\lambda_{c} w_{3}-\left(\partial w_{3} / \partial t\right)=\left(\partial w_{1} / \partial \tau\right)-\theta w_{1}+2(\partial / \partial y)\left(w_{1} w_{2}\right) \\
+e^{-t} w_{1} \int_{0}^{t} \int_{0}^{1} e^{s} w_{1}^{2}(\eta, s, \tau) d \eta d s, \\
w_{3}(0, t, \tau)=w_{3}(1, t, \tau)=0, \quad w_{3}(y, 0,0)=0 .
\end{gathered}
$$

The critical value $\lambda_{c}$ is associated with the linearized stationary version of (1.3), namely

$$
\left(d^{2} \phi / d y^{2}\right)+\lambda_{c} \phi=0, \quad \phi(0)=\phi(1)=0 .
$$


Since our interest is in the first possible onset of instability, we select the least eigenvalue for this problem, so that

$$
\lambda_{c}=\pi^{2} \quad \text { with } \quad \phi(y)=\sin \pi y .
$$

Since we shall need to solve the inhomogeneous heat equations (2.6) and (2.7) for which the homogeneous problem (2.5) has nontrivial solutions, we shall need a solvability condition. For the system

$$
\frac{\partial^{2} \psi}{\partial y^{2}}+\lambda_{c} \psi-\frac{\partial \psi}{\partial t}=r(y, t) ; \quad \psi(0, t)=\psi(1, t)=0
$$

this condition takes the form

$$
(\phi, r)=0
$$

where

$$
(a, b) \equiv \lim _{\bar{T} \rightarrow \infty} \frac{1}{\bar{T}} \int_{0}^{\bar{T}} \int_{0}^{1} a(y, t) b(y, t) d y d t .
$$

For system (2.5), condition (2.10) is trivially satisfied and the solution is given by

$$
w_{1}(y, t, \tau)=\sum_{n=1}^{\infty} A_{n}(\tau) \exp \left(-\left(n^{2}-1\right) \pi^{2} t\right) \sin n \pi y
$$

where

$$
A_{n}(0)=2 \int_{0}^{1} h(\eta) \sin n \pi \eta d \eta .
$$

Of central interest here is $A_{1}(\tau)$ since as $t \rightarrow \infty, u(y, t) \sim \varepsilon A_{1}(\tau) \sin \pi y+O\left(\varepsilon^{2}\right)$. Thus, the desired stability information by finding $A_{1}(\tau)$, which is done from the $O\left(\varepsilon^{3}\right)$ problem.

For system (2.6), the orthogonality condition takes the form

$$
\left(\sin \pi y, \frac{\partial}{\partial y}\left(w_{1}^{2}\right)\right)=0,
$$

which is automatically satisfied. Then $w_{2}$ can be obtained directly in the form

$$
w_{2}(y, t, \tau)=-\frac{1}{3 \pi} A_{1}^{2}(\tau) \sin 2 \pi y+q(y, t, \tau)
$$

where $q$ contains only terms that decay exponentially in $t$.

For system (2.7), the orthogonality condition takes the form

$$
\left.\left(\sin \pi y, \frac{\partial w_{1}}{\partial \tau}-\theta(\tau) w_{1}+2 \frac{\partial}{\partial y}\left(w_{1} w_{2}\right)+e^{-t} w_{1} \int_{0}^{t} \int_{0}^{1} e^{s} w_{1}^{2} d \eta d s\right)\right)=0 .
$$

After some manipulation, condition (2.14) becomes

$$
\frac{d A_{1}}{d \tau}-\theta(\tau) A_{1}+\frac{5}{6} A_{1}^{3}=0
$$


with

$$
A_{1}(0)=h_{1} \equiv 2 \int_{0}^{1} h(y) \sin \pi y d y .
$$

The nonlinear differential equation (2.15) is the same form as that obtained by Davis [8], DiPrima and Stuart [9], Hall [10] and Rubenfeld [11], each of whom employing different asymptotic methods in arriving at their version of this equation.

The solution of (2.15) is easily found to be

$$
A_{1}(\tau)=h_{1}\left[\frac{\exp \left(2 \int_{0}^{T} \theta(\sigma) d \sigma\right)}{1+\frac{5 h_{1}^{2}}{3} \int_{0}^{T} \exp \left(2 \int_{0}^{\sigma} \theta(\rho) d \rho\right) d \sigma}\right] 1 / 2
$$

Our stability results for the Burgers system with slow pressure fluctuations now follow from

$$
u(y, t) \sim \varepsilon\left[A_{1}(\tau) \sin \pi y+O\left(e^{-t}\right)\right]+O\left(\varepsilon^{2}\right),
$$

with the behavior of $A_{1}(\tau)$ to be determined from (2.16).

3. Stability analysis for slow pressure fluctuations. To analyze the behavior of the solution (2.17), it is convenient to introduce the notation

$$
A_{1}(\tau)= \pm\left(\frac{3}{5}\right)^{1 / 2}[y(\tau)]^{1 / 2}, \quad A_{1}(0)=h_{1}= \pm\left(\frac{3}{5}\right)^{1 / 2} y_{0}^{1 / 2},
$$

whereupon we have that $y(\tau)$ satisfies

$$
\frac{d y(\tau)}{d \tau}-2 \theta(\tau) y(\tau)+y^{2}(\tau)=0, \quad y(0) \equiv y_{0} \geq 0,
$$

whose solution is given by

$$
y(\tau)=y_{0} \frac{\exp \left(2 \int_{0}^{\tau} \theta(\sigma) d \sigma\right)}{1+y_{0} \int_{0}^{\tau} \exp \left(2 \int_{0}^{\sigma} \theta(\phi) d \phi\right) d \sigma} .
$$

Since $y \propto A^{2}$, it can be thought of as the "kinetic energy" of a velocity fluctuation whose amplitude is $A$.

Some results on the behavior of this solution have already been pointed out by Rubenfeld [11]. He has noted that

I. If $K_{1} \leq \int_{0}^{\tau} \theta(\sigma) d \sigma \leq K_{2}$, then $y(\tau) \rightarrow 0$ as $\tau \rightarrow \infty$.

II. If $\int_{0}^{\tau} \theta(\sigma) d \sigma \rightarrow-\infty$ as $\tau \rightarrow \infty$, then $y(\tau) \rightarrow 0$ as $\tau \rightarrow \infty$.

III. If $\int_{0}^{\tau} \theta(\sigma) d \sigma \rightarrow+\infty$ and $\theta(\tau) \rightarrow \theta(\infty)>0$ as $\tau \rightarrow \infty$, then $y(\tau) \rightarrow 2 \theta(\infty)$ as $\tau \rightarrow \infty$.

To these results we add the following

IV. If $\theta(\tau)=\theta_{0}+\phi(\tau)$ where $\phi(\tau)$ is $T$-periodic, $\theta_{0}>0$, and $\int_{\tau}^{\tau+T} \phi(\sigma) d \sigma=0$, then $y(\tau) \rightarrow Y(\tau)$ as $\tau \rightarrow \infty$, where $Y(\tau)$ is $T$-periodic and $T^{-1} \int_{\tau}^{\tau+r} Y(\sigma) d \sigma=2 \theta_{0}$.

V. If $\theta(\tau)=\theta_{0}+\phi(\tau)$ where $\phi(\tau)$ is quasi-periodic, $\theta_{0}>0$, and $\lim _{T \rightarrow \infty} \tau^{-1} \int_{0}^{\tau} \phi(\sigma)$ $d \sigma=0$, then $y(\tau) \rightarrow Y(\tau)$ as $\tau \rightarrow \infty$, where $Y(\tau)$ is quasi-periodic and $\lim _{\tau \rightarrow \infty} \tau^{-1} \int_{0}^{\tau} Y(\sigma)$ $d \sigma=2 \theta_{0}$. Here $\phi(\tau)$ is called quasi-periodic if $\phi(\tau) \equiv \Phi\left(\tau_{1}, \tau_{2}, \ldots, \tau_{n}\right)$, and for $j=1,2, \ldots$, $n, \tau_{j}=\tau / T_{j}$ and $\Phi$ is periodic in each $\tau_{j}$.

We thus see that, given the conditions of IV and V, periodic or quasi-periodic modulation of $\lambda$ leaves unchanged the final mean value of the "disturbance kinetic energy" $y(\tau)$. This presumes $\theta_{0}>0$; if $\theta_{0}=0$, then case I applies. 
To establish these latter results, we find it expedient to express the solution formula (3.3) as

$$
y(\tau)=y_{0} \frac{\exp \left(2 \theta_{0} \tau\right) z(\tau)}{1+y_{0} \int_{0}^{\tau} \exp \left(2 \theta_{0} \sigma\right) z(\sigma) d \sigma}, \quad z(\tau)=\exp \left(2 \int_{0}^{\tau} \phi(\sigma) d \sigma\right)
$$

First consider the case IV in which $\phi(\tau)$ is $T$-periodic. To see that $z(\tau)$ is also $T$-periodic, we note that

$$
\frac{z(\tau+T)}{z(\tau)}=\exp \left(2 \int_{\tau}^{\tau+T} \phi(\sigma) d \sigma\right)=1
$$

Next we define $Z(\tau)$ by the relation

$$
\int_{0}^{\tau} \exp \left(2 \theta_{0} \sigma\right) z(\sigma) d \sigma=\exp \left(2 \sigma_{0} \tau\right) Z(\tau)-Z(0)
$$

Since $\exp \left(2 \theta_{0} \tau\right) Z(\tau)$ is clearly a primitive for $\exp \left(2 \theta_{0} \tau\right) z(\tau)$, we can, without loss of generality, fix the value of the primitive at $\tau=0$ such that

$$
Z(0)=\left(\exp \left(2 \theta_{0} T\right)-1\right)^{-1} \int_{0}^{T} \exp \left(2 \theta_{0}\right) Z(s) d s,
$$

which always exists for $\theta_{0} T \neq 0$. Moreover, $Z(\tau)$ then has the property that

$$
Z(T)=Z(0)
$$

$Z(\tau)$ also satisfies the first-order linear equation

$$
d Z(\tau) / d \tau+2 \theta_{0} Z(\tau)=z(\tau)
$$

Having $z(\tau)=z(\tau+T)$, it follows that

$$
\frac{d Z(\tau+T)}{d \tau}+2 \theta_{0} Z(\tau+T)=\frac{d Z(\tau)}{d \tau}+2 \theta_{0} Z(\tau)
$$

The direct integration of this equation gives

$$
Z(\tau+T)-Z(\tau)=(Z(T)-Z(0)) \exp \left(-2 \theta_{0} \tau\right)=0,
$$

which confirms that $Z(\tau)$ is $T$-periodic.

It is then seen from (3.4) that

$$
y(\tau) \rightarrow Y(\tau)=z(\tau) / Z(\tau), \quad \text { as } \tau \rightarrow \infty .
$$

The mean of $Y(\tau)$ can be determined from (3.9) by considering

$$
\int_{\tau}^{\tau+T} Y(\sigma) d \sigma=\int_{\tau}^{\tau+T}\left[2 \theta_{0}+\frac{d}{d \sigma} \log Z(\sigma)\right] d \sigma=2 \theta_{0} T+\log \frac{Z(\tau+T)}{Z(\tau)}=2 \theta_{0} T .
$$

This is the desired result.

We next examine the quasi-periodic case. Under the hypothesis of case $V, \phi(\tau)$ is a 
quasi-periodic function with zero mean. Such functions can be represented by a complex series expansion of the form

$$
\phi(\tau)=\sum_{\substack{k \\(k \neq 0)}} c_{k} \exp i\left\langle k, \frac{1}{T}\right\rangle \tau .
$$

Here $k=\left(k_{1}, \ldots, k_{n}\right)$ is an $n$-vector of integers $k_{j}=0, \pm 1,+2, \ldots$, and $1 / T=\left(1 / T_{1}, \ldots\right.$, $\left.1 / T_{n}\right)$ is an $n$-vector of real numbers $1 / T_{j}>0$. Then

$$
\left\langle k, \frac{1}{T}\right\rangle=k_{1} / T_{1}+\ldots+k_{n} / T_{n}
$$

and, without loss of generality, it can be assumed that the $T_{j}$ are such that $\langle k, 1 / T\rangle=0$ if and only if $k=0$, that is, if $k_{1}=k_{2}=\ldots=k_{n}=0$. In (3.14) we have excluded $k=0$ to insure that $\lim _{\tau \rightarrow \infty} \tau^{-1} \int_{0}^{\tau} \phi(\sigma) d \sigma=0$.

Since $k=0$ has been omitted in (3.14), it follows that $\int_{0}^{z} \phi(\sigma) d \sigma$ is also a quasi-periodic function. Moreover, with reference to the solution form of (3.4), it follows that $z(\tau)$ is quasi-periodic and positive. We express it as

$$
z(\tau)=\sum_{k} d_{k} \exp i\left\langle k, \frac{1}{T}\right\rangle \tau
$$

If we again define $Z(\tau)$ as in (3.6), it follows that

$$
Z(\tau)=\sum_{k} \frac{d_{k}}{2 \theta_{0}+i\left\langle k, \frac{1}{T}\right\rangle} \exp i\left\langle k, \frac{1}{T}\right\rangle \tau,
$$

which is also a quasi-periodic and positive function.

Now from (3.4) it is seen that

$$
y(\tau) \rightarrow Y(\tau)=\frac{z(\tau)}{Z(\tau)}=\frac{\sum_{k} d_{k} \exp i\left\langle k, \frac{1}{T}\right\rangle \tau}{\sum_{k} \frac{d_{k}}{2 \theta_{0}+i\left\langle k, \frac{1}{T}\right\rangle} \exp i\left\langle k, \frac{1}{T}\right\rangle \tau}, \quad \text { as } \quad \tau \rightarrow \infty,
$$

where it is clear that $Y(\tau)$ is a quasi-periodic function.

To calculate the mean of $Y(\tau)$, we again appeal to the fact that $Z(\tau)$ satisfies the differential equation (3.9). Then, analogous, to (3.13), we find that

$$
\begin{aligned}
\lim _{\tau \rightarrow \infty} \tau^{-1} \int_{0}^{\tau} Y(\sigma) d \sigma=\lim _{\tau \rightarrow \infty} \tau^{-1} \int_{0}^{\tau}\left[2 \theta_{0}+\frac{d}{d \sigma}\right. & \log Z(\sigma)] d \sigma \\
& =2 \theta_{0}+\lim _{\tau \rightarrow \infty} \tau^{-1} \log \frac{Z(\tau)}{Z(0)}=2 \theta_{0} .
\end{aligned}
$$

Having now established IV and V, we note that the behavior outlined in I-V can now be applied to $A_{1}(\tau)$ through (3.1). This, in light of (2.18), provides results on the stability of the solution to (1.3) for various types of slow oscillations of the pressure. Under conditions I and II, we see that the disturbance velocity decays to zero. Under conditions III, IV and V, 
the disturbance velocity evolves toward a nontrivial state on the bifurcated solution branch, although in cases IV and V we see that there is oscillation about the final state. This final state has a "kinetic energy" whose mean is identical to that of the unmodulated state.

4. Stability analysis for general pressure fluctuations. In Secs. 2 and 3 we analyzed the stability of (1.3) in the special but important situation of slow pressure fluctuations about the critical value. Here we relax this restriction and examine (1.3)-(1.4) under more general circumstances to determine a condition under which we are assured that

$$
E(t) \equiv \int_{0}^{1} u^{2}(y, t) d y \rightarrow 0, \quad \text { as } t \rightarrow \infty .
$$

From (1.3)-(1.4) it is straightforward to obtain

$$
\frac{1}{2} \frac{d E(t)}{d t}=-\int_{0}^{1}\left(\frac{\partial u}{\partial y}\right)^{2} d y+\lambda(t) E(t)-e^{-t} E(t) \int_{0}^{t} e^{s} E(s) d s .
$$

By the Poincare inequality, we have

$$
\int_{0}^{1}\left(\frac{\partial u}{\partial y}\right)^{2} d y \geq \pi^{2} \int_{0}^{1} u^{2}(y, t) d y=\lambda_{c} E(t)
$$

whence follows the integro-differential inequality

$$
\frac{d E(t)}{d t} \leq 2 \Theta(t) R E(t)-2 e^{-t} E(t) \int_{0}^{t} e^{s} E(s) d s, \quad E(0) \equiv E_{0}=\int_{0}^{1} u_{0}^{2}(y) d y .
$$

Here we have defined

$$
\Theta(t)=\lambda(t)-\lambda_{c}
$$

and we hasten to note that for the case of slow fluctuations we can compare (4.6) with (2.4) to see that

$$
\Theta(t)=\varepsilon^{2} \theta\left(\varepsilon^{2} t\right) .
$$

To obtain a preliminary estimate on $E(t)$, we note that (4.5) can be simplified to

$$
d E(t) / d t \leq 2 \Theta(t) E(t), \quad E(0)=E_{0},
$$

which implies that

$$
E(t) \leq E_{0} \exp \left(2 \int_{0}^{t} \Theta(s) d s\right)
$$

To improve the estimate, we integrate (4.5) by parts to obtain

$$
\frac{d E(t)}{d t} \leq 2\left[\Theta(t)+E_{0} e^{-t}+e^{-t} \int_{0}^{t} e^{s} E^{\prime}(s) d s\right] E(t)-2 E^{2}(t), \quad E(0)=E_{0} .
$$

For the integral term in (4.10) we now utilize (4.8-4.9) to find

$$
\int_{0}^{t} e^{s} E^{\prime}(s) d s \leq 2 \int_{0}^{t} e^{s} \Theta(s) E(s) d s \leq E_{0} g(t)
$$


where

$$
g(t)=2 \int_{0}^{t} \Theta_{+}(s) \exp \left(s+2 \int_{0}^{s} \Theta_{+}\left(s^{\prime}\right) d s^{\prime}\right) d s
$$

and

$$
\begin{array}{rr}
\Theta_{+}(t)=\Theta(t), & \Theta(t) \geq 0 \\
0, & \Theta(t)<0 .
\end{array}
$$

Then (4.10) can be expressed as

$$
\frac{d E(t)}{d t} \leq 2\left\{\Theta_{+}(t)+E_{0} e^{-t}[1+g(t)]\right\} E(t)-2 E^{2}(t), \quad E(0)=E_{0} .
$$

Integration of this inequality yields

$$
E(t) \leq E_{0} \frac{\exp \left(2 \int_{0}^{t}\left\{\Theta_{+}(s)+E_{0} e^{-s}[1+g(s)]\right\} d s\right)}{1+2 E_{0} \int_{0}^{t} \exp \left(2 \int_{0}^{t} s\left\{\Theta_{+}\left(s^{\prime}\right)+E_{0} e^{-s}\left[1+g\left(s^{\prime}\right)\right]\right\} d s^{\prime}\right) d s} .
$$

To insure that (4.1) holds, it is sufficient to establish conditions under which the right side of (4.14) decays to zero as $t \rightarrow \infty$. This in turn follows from results analogous to those outlined in Sec. 3. As in Case I of those results, the desired decay in (4.14) follows if

$$
K_{1} \leq \int_{0}^{t}\left\{\Theta_{+}(s)+E_{0} e^{-s}[1+g(s)] d s \leq K_{2} .\right.
$$

Owing to the nonnegative integrand, it is clear that we can take $K_{1}=0$. To investigate the upper bound, we note that

$$
\int_{0}^{t} e^{-s}[1+g(s)] d s=\exp \left(2 \int_{0}^{t} \Theta_{+}(s) d s\right)-e^{-t}[1+g(t)] .
$$

Thus we obtain that

$$
\int_{0}^{t}\left\{\Theta_{+}(s)+E_{0} e^{-s}[1+g(s)]\right\} d s \leq \int_{0}^{t} \Theta_{+}(s) d s+E_{0} \exp \left(2 \int_{0}^{t} \Theta_{+}(s) d s\right),
$$

which motivates the condition

$$
\int_{0}^{t} \Theta_{+}(s) d s \leq m<\infty, \quad t \geq 0 .
$$

In view of (4.18) we can take $K_{2}=m+E_{0} e^{2 m}$, and thus have that the right side of (4.14) decays to zero as $t \rightarrow \infty$. Therefore, we have shown that the condition (4.18) is sufficient to provide that (4.1) holds, and thereby establish the stability of the null state.

5. Discussion. In this paper we have examined the nonlinear stability of the null state of the Burgers system. The results fall into two categories.

(i) There are sufficient conditions that the null state be stable against disturbances of arbitrary amplitude. These energy theory results hold for cases in which the modulation has 
arbitrary form but satisfies condition (4.18), i.e., for which $\Theta_{+} \rightarrow \infty$ as $t \rightarrow \infty$ sufficiently fast. Thus, there is proof of one's expectation that the ultimate reduction of forcing to a value below $\lambda_{c}$ results in stability of the null state.

(ii) The second part of the work addresses bifurcation from the null state. This bifurcation occurs at $\lambda_{c}$ and leads to supercritical equilibration consistent with the above energy theory. This behavior is then unlike that which occurs in shear flows [13] but is parallel to that in centrifugal [14] and convective [15] instabilities.

We first derive an amplitude equation (2.15) for $A_{1}$ which turns out to be the usual Landau equation but with a time-dependent coefficient. For the periodic case, similar equations $[8,9,10,11]$ have been derived for the Navier-Stokes equations. However, there have been no such derivations for quasi-periodic modulation. The new results on the behavior of the $A_{1}$ can be summarized as follows. If the pressure gradient of Burgers system consists of a mean value $P_{0}$ plus a zero-mean modulation $\tilde{P}(\tau)$ which is either periodic or quasi-periodic, then, as $t \rightarrow \infty$, the disturbance kinetic energy $y(t) \propto A_{1}^{2}(\tau)$ approaches either a periodic or quasi-periodic final state, respectively. In either case the mean value of $y$ is precisely that of the system given only the mean pressure gradient $P_{0}$. Thus, the timedependent Landau equation for $y$, which is a strongly nonlinear differential equation, behaves in this precise sense like a linear system element. The final mean of $y$ corresponds to the mean of the input $P(\tau)$. Of course, this statement does not apply to $A_{1}(\tau)$ itself.

Finally, given the work of Davis [8], DiPrima and Stuart [9], Hall [10] and Rubenfeld [11] and the present analysis, one might conjecture that the time-dependent equation

$$
d A / d \tau=\theta(\tau) A-\omega(\tau) A^{3} .
$$

might in fact be the canonical nonlinear equation emerging from bifurcation analyses of a wide variety of unsteady physical problems. The results we have obtained on the amplitude equation can be utilized here as well. If Eq. (2.16) governs Bérnard convection, then $y$ would be proportional to the convective heat transport. If Eq. (2.16) governs the instability of Couette flow between rotating cylinders, then $y$ would be proportional to the change in torque induced by instability. Our results on IV and $\mathrm{V}$ thus give the physically interesting fact that modulation leaves invariant the final mean values of the measurable integrated physical quantities. Also, it might be true that an equation of the form of (5.1) would govern bifurcation in problems involving stochastic modulation, where $\theta$ and $\omega$ would be stochastic functions. We stress however, that we have not addressed this latter problem here.

Acknowledgement. Professor S. Rosenblat made insightful comments that greatly aided this work.

\section{REFERENCES}

[1] J. M. Burgers, Mathematical example illustrating relations occurring in the theory of turbulent fluid motion, Trans. Roy. Neth. Acad. Sci. Amsterdam 17, 1-53(1939)

[2] J. M. Burgers, A mathematical model illustrating the theory of turbulence, in Advances in applied mechanics ( $R$. von Mises and T. von Kármán, editors) 1, Academic Press, New York, 171-199 (1948)

[3] G. B. Whitham, Linear and nonlinear waves, John Wiley \& Sons, 1974

[4] W. Eckhaus, Studies in nonlinear stability theory, Springer Tracts in Natural Philosophy, Vol. 6, SpringerVerlag, Berlin, 1965

[5] C. Golia and J. M. Abel, Path integral synthesis of Lyapunov functionals for partial differential equations, Int. J. Nonlinear Mech. 10, 333-334 (1975) 
[6] C. O. Horgan and W. E. Olmstead, Stability and uniqueness for a turbulence model of Burgers, Quart. Appl. Math. 36, 121-127 (1978)

[7] S. H. Davis, The stability of time-periodic flows, Ann. Rev. Fluid Mech. 8, 57-74 (1976)

[8] S. H. Davis, Finite amplitude instability of time-dependent flows, J. Fluid Mech. 45, 33-48 (1971)

[9] R. C. DiPrima and J. T. Stuart, Nonlocal effects in the stability of flow between eccentric rotating cylinders, J. Fluid Mech. 54, 393-416(1972)

[10] P. Hall, The stability of unsteady cylinder flows, J. Fluid Mech. 67, 29-64 (1975)

[11] L. A. Rubenfeld, A model bifurcation problem exhibiting the effects of slow passage through critical, SIAM J. Appl. Math. 37, 302-306 (1979)

[12] B. J. Matkowsky, A simple nonlinear dynamic stability problem, Bull. Amer. Math. Soc., 76, 620-625 (1970).

[13] W. C. Reynolds and M. C. Potter, Finite-amplitude instability of parallel shear flows, J. Fluid Mech. 27, 465-492(1967)

[14] A. Davey, The growth of Taylor vortices in flow between rotating cylinders, J. Fluid Mech. 14, 336-368 (1962)

[15] L. A. Segal and J. T. Stuart, On the question of the preferred mode in cellular thermal convection, J. Fluid Mech. 13, 289-306(1962) 